\title{
A Service Process Model for Outsourcing Human Resources Management System: Based on Massive Customized Requirements Research Perspec- tives
}

\author{
Jingyu Zhao ${ }^{1, *}$ and Xin Yan $^{2}$ \\ ${ }^{1}$ School of Management, Xiamen University, Xiamen, Fujian, 361005, P.R. China; ${ }^{2}$ China Construction Bank Inner \\ Mongolia Branch, Hohhot, Inner Mongolia, 010000, P.R. China
}

\begin{abstract}
The traditional service process model focus on personalized requirements for each customer and build model for everyone. However, when a large number of customers appear, system modeling efficiency rapidly decreased and maintenance cost rapidly increased. To solve this problem, this article proposed massive personalized requirements description service process model, MPSPM. This model firstly divides customer's personalized requirements into three dimensions: functional, efficiency, and valuable. Furthermore, in order to meet the customized modeling requirements by using minimal modeling tools, MPSPM model define the variable point and modeling standard. Finally, this article discussed a modeling case of outsourcing human resources management system. Especially develop a set of graphical modeling tools to test and verify MPSPM model.
\end{abstract}

Keywords: Massive customized requirements, service process modeling, variable point.

\section{INTRODUCTION}

In recent years, human resource management has tended to outsource model. Human resources outsourcing Services Company, like Aon Hewitt, provide a complete set of services for employers. And that service can be seen as an interaction process through provider, requester, and enabler. Customer as a service requester proposes a range of service's needs, service enablers, human resources outsourcing Services Company, negotiates different service providers and solve a solutions to customers. In the development course of modern service industry, customized requirements have been considered as vital concerns and it could bring a lot of additional values for customers. So how to satisfy customer's personalized requirements and increase the service value become a question. And how to build service model for massive customized requirements become the key to the problem proposed above.

Service model make an accurate and comprehensive description of various service elements by employing a set of standard modeling notations, and make an open and transparent communication platform for all participants involved in the service. Traditional service model focus on single customer's personalized requirements and build appropriate service model for it. Traditional service process modeling methods include the following types [1].

(1) Flow chart model. Flow chart describes the service implementation process, but does not support hierarchical description of services.
(2) Uniform Modeling Language flow chart model. It displays service process by describing the sequence of activities, and to realize the process visualization by a set of business-centric symbols.

(3) Service blueprinting model. It describes foreground events, interact with customers, and other visible elements of service in an intuitively way. But it does not describe the sequence of service activities [2, 3].

(4) Business process execution language model, BPEL. BPEL generate an execution process for multiple smallgrained web service according to specific business logic rules [4].

(5) Business process modeling notification model, BPMN. It reference a web chart, which including activities of a service and the process controller that used to define these activity execution orders. BPMN model also provide a set of notification, which can be used to build a service process model [5].

All the models reviewed above can used to build a visual service process model according to customer's personalized requirements. However, all those models have the same disadvantage: when massive customers arise, information management system needs to build a specific service solution for each customer. Therefore, service process modeling efficiency rapidly decreased, and maintenance cost rapidly increased.

Based on the discussion of massive customized requirements' characteristics, this research proposed a service process-modeling standard. This service process model can be divided into two parts. First part is built for the staff of information system. Mainly provide a wide range of service 
process modeling template and set the attributes of the customized service process elements. Second part is built for the customer. According to the classification of customer's personalized requirements, this model design different initial service templates. So the customers can use this system to customize their own service model. This article makes a theoretical contribution to the research of massive customized requirements service modeling.

\section{MASSIVE CUSTOMIZED SERVICE PROCESS MODEL}

\subsection{Description Method of Traditional Service Process Modeling}

The Among all the traditional service process models have been reviewed above, BPMN model can provide more detailed elements to describe a service process. BPMN conclude the service process modeling elements into 4 categories, flow objects, connecting objects, swim lanes, and artifacts. Where the flow objects is the core element of BPMN, which includes event, gateway, and activity [6]. We can use these visual elements to generate a service process model according to the BPMN modeling standard. For example we can use BPMN modeling standard to build an ordering service model as showed in Fig. (1).

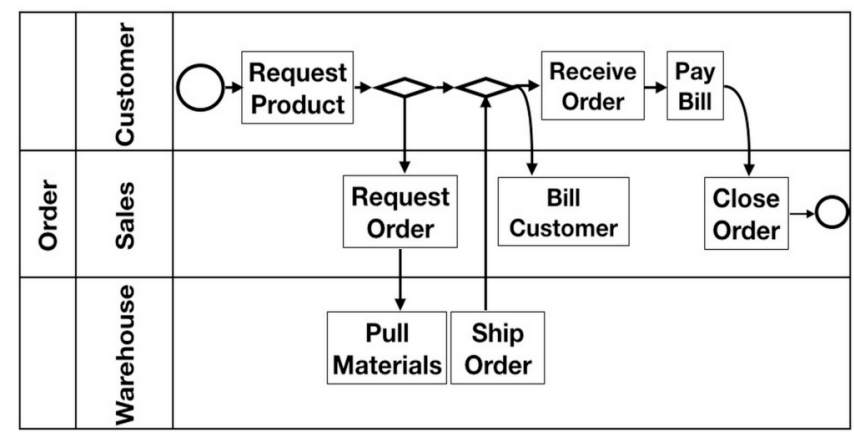

Fig. (1). An ordering service process model based on BPMN.

Fig. (1) shows a whole ordering service process model, but only for a single and basic order service process. In control flow, branching and merging only represents the relationship of activities' implementation, not represent the serv- ice process according to different customers [7]. So this model standard cannot applies to massive customized requirements service process modeling.

\subsection{Classification of Massive customized Requirements}

Customized requirements represent the personalized demand for a specific service, customer needs the service to have their own feature, and they need the service to meet their specific demands.

However, there are so many types of personalized demand in business. So we classified these demands into some standardizable and repeatable categories. In the view of customer's expectations, we classified customer's personalized demand into 3 dimensions: functional demand, efficiency demand, and valuable demand. And Table 1 shows the detail of this classification method.

Those eight types of customized requirements can be formally defined as the following equations.

(1) Service assignment. Service assignment can be defined as two categories, required task (TaskM) and optional task (TaskO). Required task refers to those tasks must be done of a specific service process. And optional task refers to those tasks whether a customer demand or not. Optional task is a part that customer can determined by themselves.

$$
\text { Task }=\left(T_{-} I D, T_{-} \text {Name, } T_{-} \text {Type, } T_{-} \text {Choose }\right)
$$

in where, $T_{-} I D$ refers to assignment ID; $T_{-}$Name refers to the assignment name; $T_{-}$Type refers to the assignment type, $T_{-}$Type $\in\left[\right.$ TaskM,TaskO]; $T_{-}$Choose refers to customer's choice, $T_{-}$Choose $\in[0,1]$.

(2) Resources. Service quality can be determined by service resource input. And customers have their personal preference about the price and quantity of service resource.

$$
\text { Task_Res }=\left(R_{-} I D, R_{-} \text {Name, } R_{-} \text {Type, } R_{-} \text {Num, } R_{-} \text {Pr }\right)
$$

in where, $R_{-} I D$ refers to resource ID; $R_{-}$Name refers to the resource name; $R_{-}$Type refers to the resource type, $R_{-}$Num refers to the resource quantity; $R_{-} \operatorname{Pr}$ refers to the resource price.

\begin{tabular}{|c|c|c|}
\hline Dimensions & Subcategories & Description \\
\hline \multirow{3}{*}{ Functional demand } & Service assignment & Options that customers may have during a service process \\
\hline & Resources & Preference about the resource and price \\
\hline & Sequence of assignments & Preference about the service sequence in a service process \\
\hline \multirow{2}{*}{ Efficiency demand } & Assignment QoS & Preference about the efficacy and quality of one or several service assignments \\
\hline & Service QoS & Preference about the efficacy and quality of one or several service property \\
\hline Valuable demand & Observable benefits and it's constraints & Customer's preference about the observable benefits and it's constraints \\
\hline \multirow{2}{*}{ Valuable demand } & Observable cost and it's constraints & Customer's preference about the observable cost and it's constraints \\
\hline & Latent benefits and it's constraints & Customer's preference about the latent benefits and it's constraints \\
\hline
\end{tabular}

Table 1. Result of experiments and theory. 


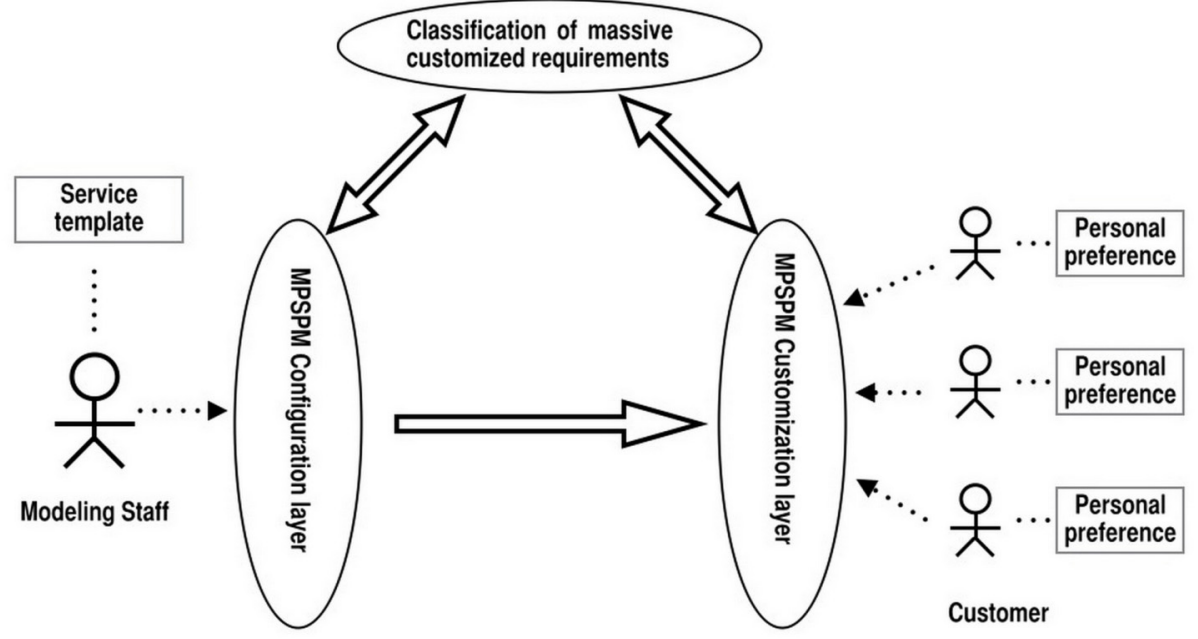

Fig. (2). Framework of service process model.

(3) Sequence of assignments. The sequence of assignments can be determined by customer's personalized preferences. And the sequence of assignments also determines the pattern of a service process model.

Task_Task $=\left(T_{-}\right.$IDset, $T_{-} T_{-}$Choose $)$

in where, $T_{-}$IDset refers to a set of assignments; $T_{-} T_{-}$Choose refers to customer's personalized assignments sequence.

(4) Assignment QoS. The quality of each assignment can affect the whole service quality evaluation. However customer have different preference to a specific assignment.

$$
\text { Task_QoS }=\left(\text { QoS } S_{-} I D, Q o S_{-} \text {Opt,QoS_Choose }\right)
$$

in where, QoS $I D$ refers to assignment quality ID; QoS_Opt refers to configurable intervals of a specific assignment QoS; QoS_Choose refers to customer's configuration interval, QoS_Choose $\in$ QoS_Opt.

(5) Service QoS. Compared to assignment QoS, Service QoS focus on the whole quality and efficiency of a service process.

Service_QoS $=\left(Q o S_{-} I D, Q o S_{-} O p t, Q o S_{-}\right.$Choose $)$

in where, $Q o S_{-} I D$ refers to service quality ID; QoS_Opt refers to configurable intervals of a specific service QoS; QoS_Choose refers to customer's configuration interval, QoS_Choose $\in$ QoS_Opt.

(6) Observable benefits and it's constraints. Observable benefits and its constraints can both affect the service quality and customer's service experience. The constraint is determined by customer's preference.

$$
B_{-} C O N B=\left(B, B_{-} Q \text { Name, } B_{-} \text {QValue, } B_{-} \text {QWeight }\right)
$$

in where, $B$ refers to observable benefit of a specific assignment; $B_{-}$QName refers to the name of constraints about a specific observable benefit; $B_{-}$QValue refers to the value of a specific constrains; $B \_Q W e i g h t$ refers to weight coefficient of a specific benefit quality.

(7) Observable cost and it's constraints. Observable costs and its constraints directly affect the service quality and resource input of a specific service process. Cost constraint is determined by customer's preference.

$$
C_{-} \text {CONC }=\left(C, C_{-} \text {QName, } C_{-} \text {QValue, } C_{-} Q \text { Weight }\right)
$$

in where, $C$ refers to observable cost of a specific assignment; $C_{-}$QName refers to the name of constraints about a specific observable benefit; $C_{-} Q$ Value refers to the value of a specific constrains; $C_{-} Q W e i g h t$ refers to weight coefficient of a specific benefit quality.

(8) Latent benefits and its constraints. Latent benefits are determined by customer's expectations and the constraints of resources. Also latent benefits can be customized.

$E_{-} C O N E=\left(E, E_{-} Q N a m e, E_{-} Q\right.$ Value,$E_{-} Q$ Weight $)$

in where, $E$ refers to latent benefit of a specific assignment; $E_{-}$QName refers to the name of constraints about a specific latent benefit; $E_{-}$QValue refers to the value of a specific constrains; $E_{-} Q$ Weight refers to weight coefficient of a specific benefit quality.

\section{SERVICE PROCESS MODEL}

Based on the review and theory analysis above, we introduce a new service process modeling method, MPSPM. MPSPM is based on customer's personalized demand classification, following the description of configuration layer and customization layer. This modeling method provides a unified concept of environment for everyone that participated in service process.

We divided MPSPM into configuration layer and customization layer in order to fit massive customized requirements. Configuration layer is build for modeling staff. Modeling staff can set the basic service template and the relevant variable location. Customization layer is build for customer. 
Customer can set their personalized preference on the specific template that has been generated by configuration layer. Finally a customized service model can be automatically generated from the template that has been modified by customer. And Fig. (2) shows that model framework.

\subsection{Elements of MPSPM Model}

The description of MPSPM is based on an assignmentcentric way. Use graphics to express service assignments, service elements, and assignment sequence. Use implicit equation to express assignment property information. Based on the definition and description of service process model, main elements of MPSPM include: swim lanes, service assignments, service quality requirements and so on. And Table 2 shows each element's graphics primitive and attribute description.

Table 2. Elements of MPSPM model.

\begin{tabular}{|c|c|c|}
\hline Elements & Graphics Primitive & Description \\
\hline \multirow{2}{*}{ Swim Lane } & Customer & \multirow{2}{*}{ Swim Lane } \\
\hline & \begin{tabular}{|l|l|}
$R L, 1.1$ & $R L, 1.2$ \\
\end{tabular} & \\
\hline Task & $\begin{array}{l}\text { <Interactive tasks M> } \\
\text { ITaskM }\end{array}$ & Service task \\
\hline VP & $\begin{array}{l}\text { I Variable Point I } \\
\text { I VP }--- \text { I }\end{array}$ & Variable point \\
\hline QR & QR & $\begin{array}{l}\text { Service quality } \\
\text { required }\end{array}$ \\
\hline PCL & & $\begin{array}{l}\text { Process connec- } \\
\text { tion line }\end{array}$ \\
\hline T-TS & $-\quad-$ & $\begin{array}{l}\text { Task relation- } \\
\text { ship links }\end{array}$ \\
\hline
\end{tabular}

Table 2 show the elements of MPSPM model, and the attribute set of these elements are as follows. Swimlane $=($ info, type $) ;$ Task $=($ info, Res, B, C, E, CONS $)$; $V P 1=$ (Task,Task_Res, Task_Task,Task_QoS, Task_Service); $V P 2=($ Task_B,Task_C,Task_E $) ; P C L=($ info, SO,EO,CONS $)$ $Q R=\left(Q O S \_I D, Q o S_{-}\right.$Name, Qos_Value $) ; T_{-} T s=($ info, $S O, E O)$. And all the elements including the model must obey the attribute set.

\subsection{MPSPM Configuration Layer}

In order to support customized requirements, we firstly define the concept of variable point as following. Variable point refers to the service elements that customer can determine to satisfy their demand during a service execution process. And variable point can be express as following equation.
$V P=\left(V P\right.$ Position, $V P_{-}$Value, VP_Expreession)

in where, VP_Position refers to the variable point position

VP_Position $\in\{$ Task,Task_Res,Task_Task,Task_QoS,Servic e_Qos, $\left.B_{-} C O N B, C_{-} C O N C, E_{-} C O N E\right\}$. VP_Value refers to the service element' value of a specific variable position. VP_Expreession refers to the service element's expression of a variable point with customer's configuration.

Firstly, about MPSPM configuration layer modeling rules. The modeling rules are as follows.

Rule \#1 if VP_Position $=\{$ Task_Res or Task_QoS or $B C O N B$ or $C \overline{C O N C}$ or $E C O \overline{N E}\}$, then staff can configure a lot of variable positions, process connector's start object corresponding to service assignment.

Rule \#2 if VP Position= Task, then Task= TaskO. It means the customized service process is a template standardization service process, and that service model can be choosing from the database. Process connector object should be the beginning of a variable point.

Rule \#3 if VP Position = Task_Task, let T TS connect all the service assignments. Process connector object should be the beginning of a variable point.

Rule \#4 if VP_Position = Service_Qos, then set related service quality variable position to the customized value. Process connector object should be connecting to QR graphics primitive.

Secondly, MPSPM configuration layer modeling process. The modeling processes are as follows.

Step 1. Build the template based on SPC view modeling rule, which is the core of SMPD service modeling standard.

Step 2. Introduce variable point graphics primitive into model corresponding to customer's personalized demand.

Step 3. Fill correlated information into the box.

Step 4. Configure the variable point based on rule\#1, rule $\# 2$, rule\#3, and rule\#4 that has been defined above.

\subsection{MPSPM Customization Layer}

MPSPM Configuration layer is primarily designed for customers. Customers can set their preference on the service process template that been generated by MPSPM configuration layer.

Firstly, MPSPM customization layer modeling rules are as following.

Rule \#1 all Task_ID must be the same for one service model.

Rule \#2 about the value set of Service_QoS and Task_QoS. The value of $Q o S$ in Customization layer must be a subset of $Q O S$ in configuration layer.

Rule \#3 Use "A-B-C" mode to describe the assignments ID which is included in the set of service assignments. What's more, use "1-2-3" mode to describe the sequence of assignments.

Secondly, MPSPM customization layer modeling process 


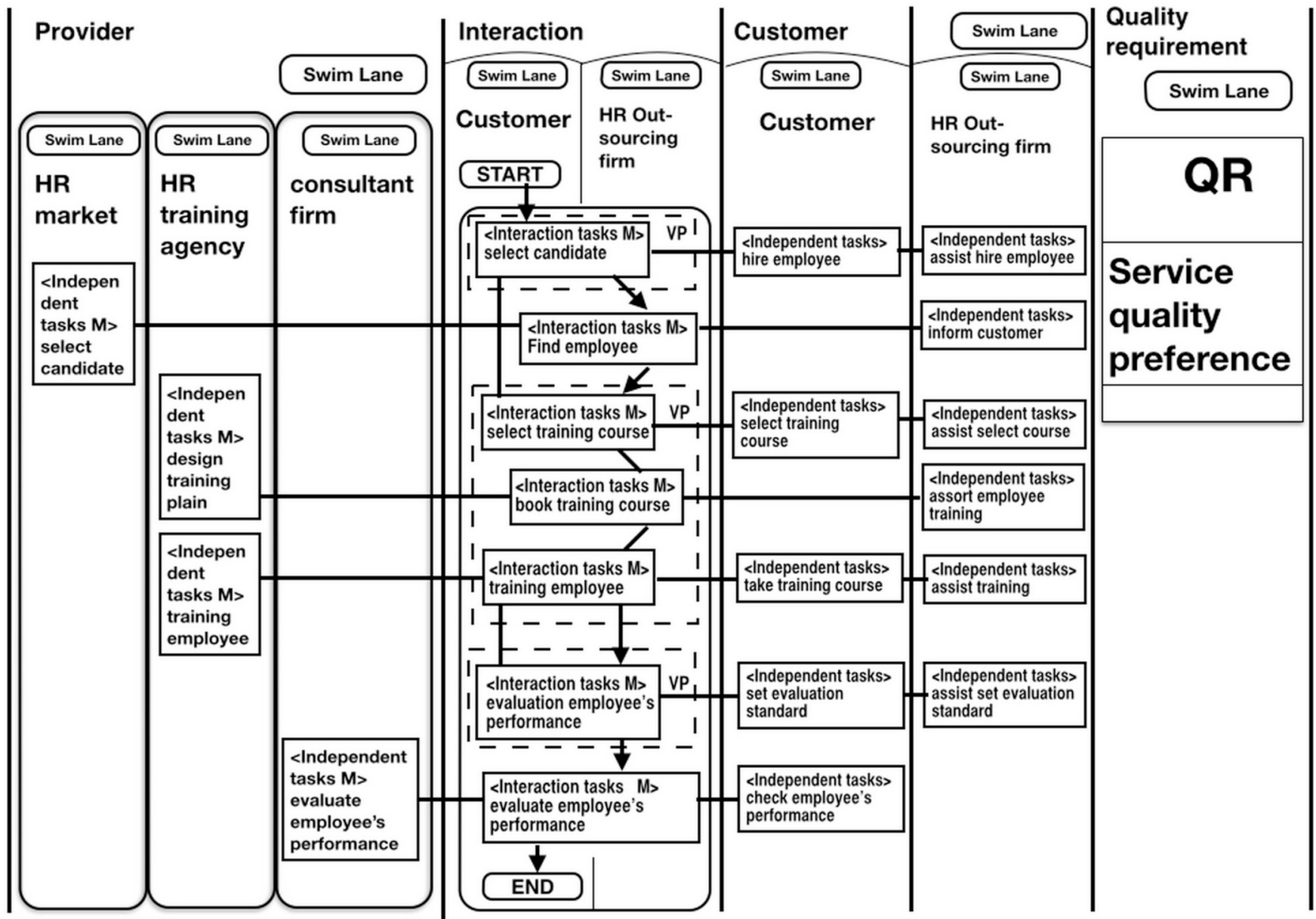

Fig. (3). A service process model template based on MPSPM.

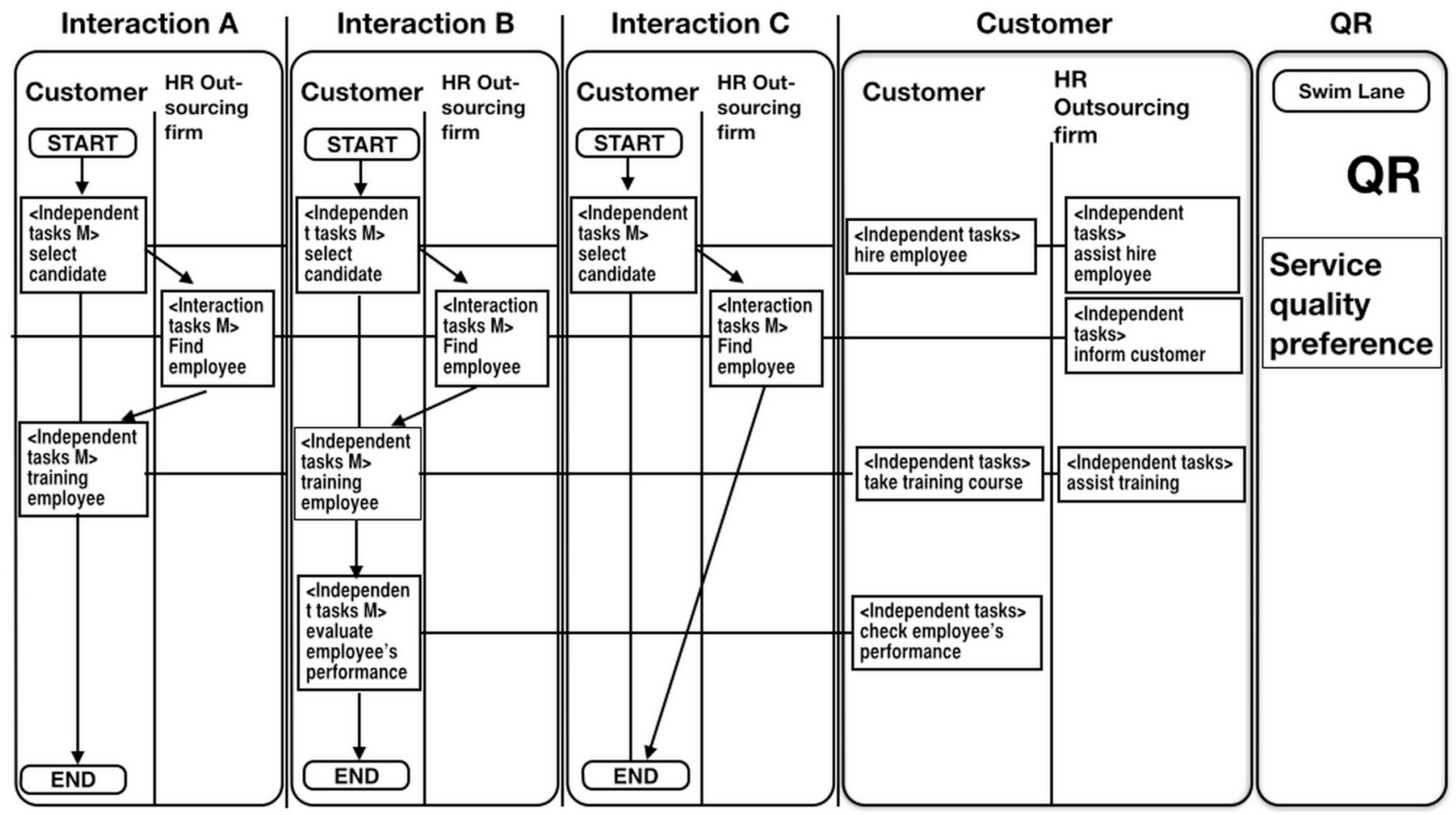

Fig. (4). An outsourcing human resources service process mode. 
Step 1. Based on the template, let customer use the preference menu to set their service preference.

Setp 2. Use "generate service" menu to generate the customized service process model.

\section{A CASE STUDY}

Outsourcing human resources is becoming a trend for companies to manage their HR resources. It takes a lot of convenient and strategy advantage for customer. But outsourcing human resources management firm has to face many of customers, each of them has their own requirements. Traditional service modeling method cannot satisfy this situation. This section will use the modeling method that been discussed above, give a demonstration of service process modeling.

Assignments of an outsourcing human resources service include: proposed employment requirements, training, and performance evaluation. Resources of an outsourcing human resources service include: employee, training skills, and performance evaluation consulting resources [8]. Finally, Fig. (3) shows a service process model template, and Fig. (4) shows a whole customized service model.

From the service process model template and customized service model above, we can find that MPSPM can build service process model for each customer's personalized requirements and shows the advantage of operational convenient

\section{CONCLUSION}

From the case study we can find that MPSPM modeling method is an effective modeling way for massive customized requirements. Both service provider and customer can achieve service value form the convenient modeling process. We designed MPSPM have two layer, configuration layer and customization layer. This design solved the problem of maintenance cost rapidly increased when there is so many customers propose personalized demands.

Though MPSPM fill a theory gap of massive customized requirements, there are still some limits about this research.
The different set of demand classification will affect modeling efficiency. This article only discussed one classification method [9]. That is one of the limits of this research. What's more, this research only discussed one simple case. And we believe MPSPM model can also apply to other service patterns. Above all, further research can focus on the different personalized demand classification method. And the modeling standard also needs more study.

\section{CONFLICT OF INTEREST}

The authors confirm that this article content has no conflict of interest.

\section{ACKNOWLEDGEMENTS}

Both author wish to thanks the advice from Dr. Ma Zhuang and Dr. Han haitao.

\section{REFERENCES}

[1] R. S. Aguilar-Saven, "Business process modelling: Review and framework", International Journal of Production Economics, vol. 90, pp. 129-149, 2004

[2] G. L. Shostack, "How to design a service", European Journal of Marketing, vol. 16, pp. 49-63, 1982.

[3] M. J. Bitner, A. L. Ostrom, and F. N. Morgan, "Service blueprinting: A practical technique for service innovation", California Management Review, vol. 50, pp. 66-90, 2008.

[4] M. B. Juric, B. Mathew, and P. G. Sarang, Business Process Execution Language for Web Services: An Architect and Developer's Guide to Orchestrating Web Services Using BPEL4WS, Packt Publishing Ltd, 2006, pp. 55-65.

[5] A. L. N. Campos, and T. Oliveira, "Software processes with BPMN: an empirical analysis", In: Product-Focused Software Process Improvement, Springer, Berlin Heidelberg, 2013, pp. 338-341.

[6] R. Dijkman, J. Hofstetter, and J. Koehler, Eds., Business Process Model and Notation, Springer, 2011, pp. 10-12.

[7] P. Wohed, On The Suitability of BPMN for Business Process Modelling, Springer, Berlin Heidelberg, 2006, pp. 20-26.

[8] N. P. Kumar, "Human resource management in future an obstacle of champion of globalization", Human Resource Management, vol. 1, no. 1, 2014, pp. 49-57.

[9] M. J. Jahid, T, H. Huang, and J. Ruan, "A personalized committee classification approach to improving prediction of breast cancer metastasis", Bioinformatics, vol. 30, no. 13, 2014, pp. 1858-1866.

Received: June 10, 2015

Revised: July 29, 2015

Accepted: August 15, 2015

(C) Zhao and Yan; Licensee Bentham Open.

This is an open access article licensed under the terms of the (https://creativecommons.org/licenses/by/4.0/legalcode), which permits unrestricted, noncommercial use, distribution and reproduction in any medium, provided the work is properly cited. 